\title{
The place of D-dimer and L-lactate levels in the early diagnosis of acute mesenteric ischemia
}

\author{
Aydin $\mathrm{B}^{1}$, Ozban $\mathrm{M}^{2}$, Serinken $\mathrm{M}^{3}$, Kaptanoglu $\mathrm{B}^{4}$, Demirkan $\mathrm{NC}^{5}$, Aydin $\mathrm{C}^{2}$ \\ Anadolu Saglik Merkezi Hospital, Department of Emergency Medicine, Denizli, Turkey. \\ muratozban@yahoo.com
}

\begin{abstract}
INTRODUCTION: Acute mesenteric ischemia (AMI) is an abdominal-vascular emergency which is rare and has high mortality rates $(60-80 \%)$ due to late diagnosis (1-3). Although it is known that extravascular reasons like intestinal intussusception, volvulus, strangulated hernias and obstructions can cause intestinal gangrene, these are rarely the cause of AMI (1).

MATERIALS AND METHODS: In this study, we used male Wistar-Albino rats weighing 250-300 grams obtained from Pamukkale University Experimental Research Laboratory. Animals were exposed to light-dark cycles for 12 hours and had free access to food and water. They were kept in cages for 7 days to stabilise their intestinal flora. In animals of group I, nothing was made other than taking $0.5 \mathrm{ml}$ blood intracardially. In other animals, abdomen was reached with midline laparotomy and superior mesenteric artery (SMA) was located. In group II (operative control group), SMA was isolated and manipulated but was not ligated. In Group III (intestinal ischemia group), SMAwas isolated and ligated with $3 / 0$ silk tie distally to the aorta. After this process, intestinal ischemia was achieved which was confirmed by paleness and pulselessness of intestines, caecum and right colon. Later on, abdomen was closed with double $3 / 0$ polyglactin sutures. At postoperative $1 \mathrm{st}$, 4 th and 6 th hours $0.5 \mathrm{ml}$ blood was taken intracardially from the animals in groups II and III in order to quantify D-dimer and L-lactate levels. LABORATORY TESTS: D-dimer: Blood samples which were put into tubes containing sodium citrate, were seperated from plasma with centrifugation at $4000 \mathrm{rpm}$ for 7 minutes.

L-lactate: Blood L-lactate levels were determined from blood taken into capillary tubes with the help of immobilised enzyme electrode technology using YSI 1500 Sport portative lactate analyzer (Yellow Springs Instruments Inc., Ohio-USA).

HISTOPATHOLOGIC VERIFICATION: Two $\mathrm{cm}$ long intestinal samples were taken from animals in which SMA was ligated in order to achieve mesenteric ischemia and these samples were fixed in $10 \%$ formol.

DISCUSSION: As a result, in rats with SMA occlusion serum D-dimer levels were not increased significantly when compared either in the group or with the basal values of the control group and values in operative control group. Therefore, it is concluded that D-dimer is not a useful marker for early diagnosis of AMI. On the other hand, it is revealed that blood L-lactate levels began to increase significantly following 4th hour of mesenteric ischemia and it is shown that this increase continued at the 6th hour. In addition, considering the utmost importance of the early diagnosis in patients with the clinical suspicion of AMI, L-lactate seems to be a suitable marker to use in emergency departments because it is achieved with a portable device that gives fast and accurate results. Nevertheless, our results are need to be supported by clinical studies with larger patient series (Tab. 2, Fig. 11, Ref. 39). Text in PDF www.elis.sk.

KEY WORDS: D-dimer, L-lactate, acute mesenteric ischemia.
\end{abstract}

\section{Introduction}

Acute mesenteric ischemia (AMI) is an abdominal-vascular emergency which is rare and has high mortality rates $(60-80 \%)$ due to late diagnosis (1-3). AMI is seen in one out of thousand but

\footnotetext{
${ }^{1}$ Anadolu Saglik Merkezi Hospital, Department of Emergency Medicine, ${ }^{2}$ Pamukkale University, School of Medicine, Department of General Surgery, ${ }^{3}$ Pamukkale University, School of Medicine, Department of Emergency Medicine, ${ }^{4}$ Pamukkale University, School of Medicine, Department of Biochemsitry, and ${ }^{5}$ Pamukkale University, School of Medicine, Department of Pathology
}

Address for correspondence: M. Ozban, MD, Pamukkale University, School of Medicine, Department of Surgery, Denizli, Turkey Phone: +90.258 .4440728$ the incidence is increasing (4). Although it is known that extravascular reasons like intestinal intussusception, volvulus, strangulated hernias and obstructions can cause intestinal gangrene, these are rarely the cause of AMI (1). The etiology of AMI can be evaluated in 4 sub-categories: superior mesenteric artery (SMA) embolism (45-50\%), thrombosis of SMA (25\%), superior mesenteric vein (SMV) thrombosis (5-10\%), and non-occlusive mesenteric ischemia (NOMI) (20\%) (4-7). Despite the differences in its etiology, intestinal gangrene and necrosis is the fatal end of AMI $(3,7)$.

Despite the improvements at diagnostic imaging methods, surgical techniques and perioperative intensive care support, the high mortality rate due to AMI did not change significantly in the last seven decades $(5,7)$. Reason of this are the ongoing difficulties at establishing AMI diagnosis before bowel necrosis occurs 
(7). In a review of Herbert et al, it is reported that only one third of the patients was diagnosed as AMI before the surgical exploration and death (5). In Swedish autopsy studies, it was shown that real mortality rate from mesenteric ischemia can be more than $90 \%$, and $22-32 \%$ of these patients could be diagnosed before death $(8,9)$. It is known that delays at establishing the AMI diagnosis reduce the survival chance and morbidity (4). Patient presentation is usually non-spesific and normal physical examination of the abdomen can cause a dangerous safety feeling of the physician $(4,5)$.

For early diagnosis of AMI, recent radiological imaging methods play a limited role. X-rays are non-specific and are used to exclude reasons of acute abdomen like perforated peptic ulcus. Computerized tomography (CT) can show thickening of bowel walls, ascites or mesenteric artery obstruction but these all are non-specific findings which can be seen in the late stages of intestinal ischemia (3). Even though Doppler ultrasonography is useful in complete obstruction of proximal SMA, it is suboptimal because it relies on the expertness of the practitioner (4). Selective mesenteric angiography is considered the golden standart of AMI diagnosis but it is not available at every hospital, it requires an interventional radiologist, it is an invasive and long procedure and it requires nephrotoxic agents (3-5).

Unfortunately, there are no laboratory tests which can be used in early diagnosis of AMI properly. Even though serum amylase, aspartate aminotransferase (AST), lactate dehydrogenase (LDH) and creatine phosphokinase (CPK) are elevated, none of these are sensitive or specific (7). There are a lot of studies investigating the serum levels of D-dimer, alpha-glutathion S-transferase, Dlactate, L-lactate, $\mathrm{LDH}$, intestinal fatty acid binding protein, alkaline phosphatase and CPK for early diagnosis of AMI but there are controversial reports about the usage of these markers in early diagnosis of instestinal ischaemia (10-14).

D-dimer is a fibrin degradation product and it can rise in conditions like disseminated intravascular coagulation (DIC), deep vein thrombosis and pulmonary embolism as a stable component of the fibrinolytic system $(10,12,15,16)$. L-lactate is constantly produced from pyruvate via the enzyme lactate dehydrogenase (LDH) during anaerobic glycolysis (14). Clinically, it is reported that lactate levels are elevated in $90 \%$ of AMI patients and these levels are associated with mortality (4). In an experimental study, significant elevation of L-lactate levels were seen in rats with SMA occlusion (16). In another experimental study, there was no significant elevation of L-lactate levels after SMA occlusion (11). The aim of this study was to investigate serum D-dimer and L-lactate levels in rats with superior mesenteric artery occlusion model.

\section{Materials and methods}

In this study, we used male Wistar-Albino rats weighing 250300 grams obtained from Pamukkale University Experimental Research Laboratory. Animals were exposed to light-dark cycles for 12 hours and had free access to food and water. They were kept in cages for 7 days to stabilise their intestinal flora. Before the study, consent was taken from the Pamukkale University Medical Faculty Experimental Studies Ethics Committee and all operative procedures, usage of anaesthesia and animal care processes were done according to the universally accepted guidelines (National Institutes of Health Guidelines on the Care and Use of Laboratory Animals, NIH publication No.86-23, revised 1985, Bethesda, Maryland, ABD).

Animals were randomised into 3 groups which had 10 animals in each:

Group I: Non-operative control group: In these animals, only blood was taken to spot basal D-dimer and L-lactate levels.

Group II: Operative control group: In these animals, no blood was taken before the surgical procedure. SMA was identified and manipulated elementarily after the laparotomy.

Group III: Intestinal ischemia group: In these animals, intestinal ischemia was created by SMA ligation after laparotomy.

\section{Operational details}

After fasting over night, animals were anesthetized using 50 $\mathrm{mg} / \mathrm{kg}$ intramuscular ketamine (Ketalar; Eczacibasi Ilac ve Ticaret A.S. Istanbul - Turkey) and $10 \mathrm{mg} / \mathrm{kg}$ xylazine (Rompun; Bayer Türk Kimya San. Ltd. Sti. Istanbul - Turkey). During surgical procedure, spontaneous ventilation of the rats was ensured. A heater lamp was used to keep body temperature at $37^{\circ} \mathrm{C}$. In order to prevent dehydration after the surgery, $10 \mathrm{ml}$ Ringer lactate solution was used subcutaneously. After shaving, abdominal area was wiped using $10 \%$ povidone iodine twice and surgery began with sterile equipment. In animals in group I, nothing was made other than taking $0.5 \mathrm{ml}$ blood intracardially. In other animals, abdomen was reached with midline laparotomy and superior mesenteric artery (SMA) was located.Int Group II (operative control group), SMA was isolated and manipulated but not ligated. In Group III (intestinal ischemia group), SMA was isolated and ligated with $3 / 0$ silk tie distally to the aorta. After this process, intestinal ischemia was achieved which was confirmed by paleness and pulselessness of intestines, caecum and right colon. Later on, abdomen was closed with double $3 / 0$ polyglactin sutures. At postoperative 1st, 4th and 6th hours $0.5 \mathrm{ml}$ blood was taken intracardially from the animals in groups II and III in order to quantify D-dimer and L-lactate levels. At the end of the experiment, all animals were killed by excessive anaesthesia and relaparotomy was performed to get intestinal samples. Abominal findings were noted.

\section{Laboratory tests}

D-dimer: Blood samples which were put into tubes containing sodium citrate, were seperated from plasma with centrifugation at $4000 \mathrm{rpm}$ for 7 minutes. Immunoturbidimetric assay of fibrin split products (D-dimer) was performed using Innovance ${ }^{\circledR}$ D-dimer kit and BCS XP System ${ }^{\circledR}$ coagulation analyser (Dade Behring, Marburg-Germany). In this method, normal plasma D-dimer levels are considered $<0.5 \mathrm{mg} / \mathrm{L}$ FEU.

L-lactate: Blood L-lactate levels were determined from blood taken into capillary tubes with the help of immobilised enzyme electrode technology using YSI 1500 Sport portative lactate analyzer (Yellow Springs Instruments Inc., Ohio-USA). In this technology, hydrogen peroxide that comes up proportionally to lactate, which has been diffused after passing of the blood sample from 


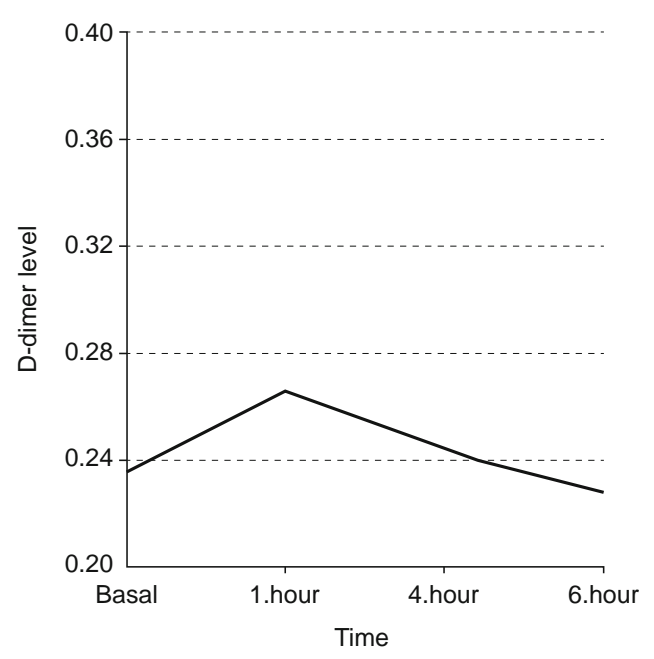

Fig. 1. Post-op D-dimer levels at group II ( $p=0,584$, Friedman variance analyse).

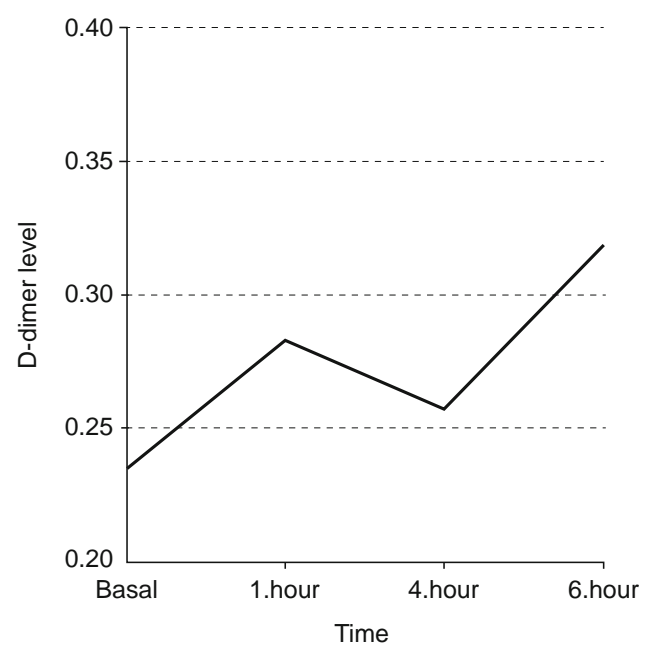

Fig. 2. Post-op D-dimer levels at group III ( $p=0.717$, Friedman variance analyse).

an enzyme-membrane system, is quantified on a platinum anode. Results are displayed as $\mathrm{mmol} / \mathrm{L}$. Twenty-five $\mu \mathrm{L}$ blood is enough for the measurement. Results are acquired within 60 seconds in automatic calibration mode.

\section{Histopathologic verification}

Two $\mathrm{cm}$ long intestinal samples were taken from animals in which SMA was ligated in order to achieve mesenteric ischemia and these samples were fixed in $10 \%$ formol. Materials were embedded in paraffin, sections at $5 \mu \mathrm{m}$ were stained with haematoxylin-eosin and examined under a light microscope.

\section{Statistical assessment}

Data management and statistical analyses were done using SPSS for Windows, version 11.5 (SPSS Inc., Chicago, Illinois, USA). In groups II and III, intergroup variation comparison of

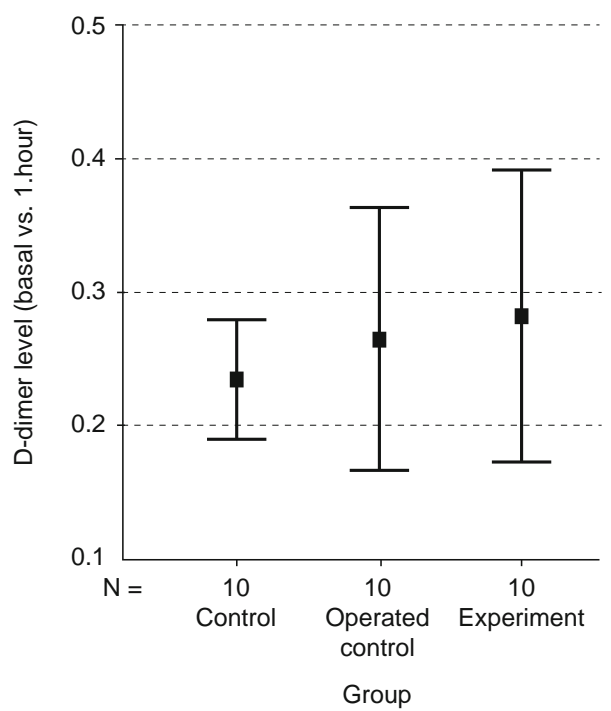

Fig. 3. Kruskal-Wallis variance analyse $(p=0.703)$.

D-dimer and L-lactate measurements were done using Friedman variation analysis at different time points and $\mathrm{p}<0.05$ was considered significant. Results found to be statistically significant with this test proceeded to post hoc analysis with Wilcoxon test. Moreover, results between group I and groups II and III were compared using Kruskal-Wallis variation analysis and post hoc evaulation beetween groups were done using Mann-Whitney U test.

\section{Results}

Operative findings

During the perioperative period, 2 rats from superior mesenteric artery occlusion group (Group III) died. One of these rats died in the first hour of ischemic process, and the other one died at the 4th hour of ischemic process. Two new rats were included into the study to replace these rats. Relaparotomy after sacrificing all animals with excessive anaesthesia usage revealed that rats in non-operative control group (Group I) and operative control group (Group II) had normal intestines at inspection. In contrast, intestines of the rats in group III were dilated, edematous and obviously cyanotic.

\section{D-dimer}

In order to identify if there is a rising tendency in D-dimer levels at postoperative 1st, 4 th and 6 th hour in groups II and III, Friedman test was conducted. There was no significant rising tendency in the operative control group (Group II) $(p=0.584)$ (Fig. 1). Rats who have mesenteric ischemia (Group III - experimental group) had a minor increase in D-dimer levels at sixth hour but this was not statistically significant ( $p=0.717$ ) (Fig. 2).

In addition, Kruskal-Wallis variation analysis was conducted in order to compare D-dimer levels at 1st, 4th and 6th hour in Groups II and III with basal D-dimer level of group I. There was no significant difference between basal D-dimer levels and levels at 1 hour ( $p$ 


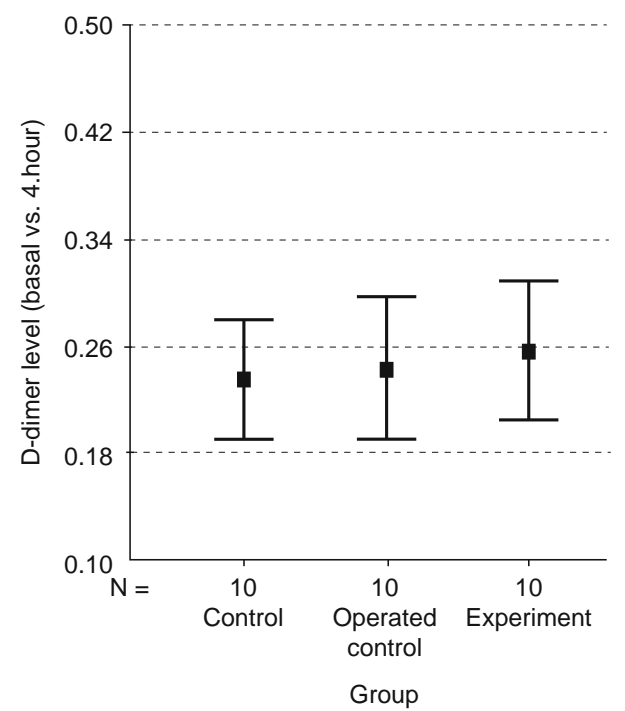

Fig. 4. Kruskal-Wallis variance analyse $(\mathrm{p}=\mathbf{0 . 6 4 6})$.

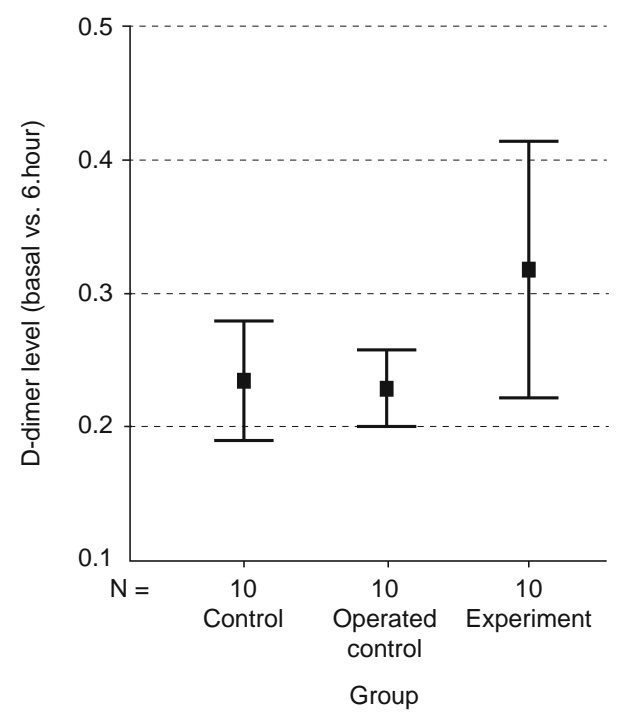

Fig. 5. Kruskal-Wallis variance analyse $(p=0.061)$

$=0.703)$ (Fig. 3) observed. Similarly, there was no significant difference between basal level and levels at 4 and 6th hours in groups II and III ( $\mathrm{P}=0.646$ vs $\mathrm{P}=0.061$, respectively) (Figs 4 and 5). Mean basal D-dimer levels of group I and mean D-dimer levels at 1,4 , and 6 . hour of group II and III can be found in Table 1.

\section{L-Lactate}

Friedman test was conducted in order to identify if there is a rising tendency in L-lactate levels which were evaluated at postoperative 1st, 4th and 6th hour in groups II and III. There was no significant rising tendency in the operative control group (Group II) $(p=0.097)$ (Fig. 6) observed. In the rats with mesenteric ischemia (Group III), mean L-lactate levels began to rise at fourth hour and this increase continued at 6 th hour $(p=0.0001)$ (Fig. 7). In this
Tab. 1. Mean D-dimer levels.

\begin{tabular}{lcccc}
\hline D-dimer & $\begin{array}{c}\text { Group I } \\
(\text { mean } \pm \text { SD })\end{array}$ & $\begin{array}{c}\text { Group II } \\
(\text { mean } \pm \text { SD })\end{array}$ & $\begin{array}{c}\text { Group III } \\
(\operatorname{mean} \pm \text { SD })\end{array}$ & p \\
\hline basal & $0.23 \pm 0.06$ & - & - & \\
1st hour & - & $0.26 \pm 0.13$ & $0.28 \pm 0.15$ & $=0.703$ \\
4th hour & - & $0.24 \pm 0.07$ & $0.25 \pm 0.07$ & $=0.646$ \\
6th hour & - & $0.22 \pm 0.04$ & $0.31 \pm 0.13$ & $=0.061$ \\
\hline
\end{tabular}

group, paired comparison of mean L-lactate levels at 1st, 4th and 6 th hours were conducted using Wilcoxon test and $\mathrm{p}<0.017$ was determined to be significant. According to this, L-lactate levels at 4 th hour rised significantly when compared with first hour $(\mathrm{p}=$ 0.005 ), and this rise was still significant at 6th hour when compared with 4th hour levels ( $\mathrm{p}=0.007)$.

Kruskal-Wallis variation analysis was conducted in order to

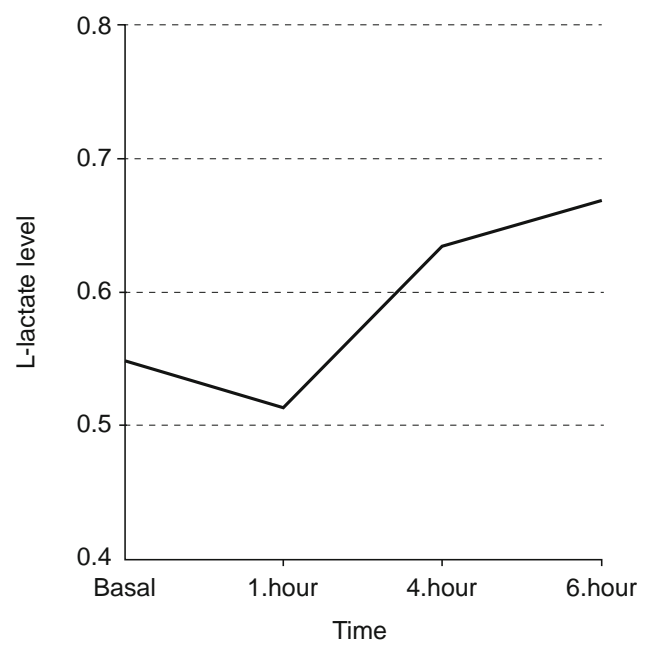

Fig. 6. Post-op L-lactate levels at group III ( $p=0.097$, Friedman variance analyse).

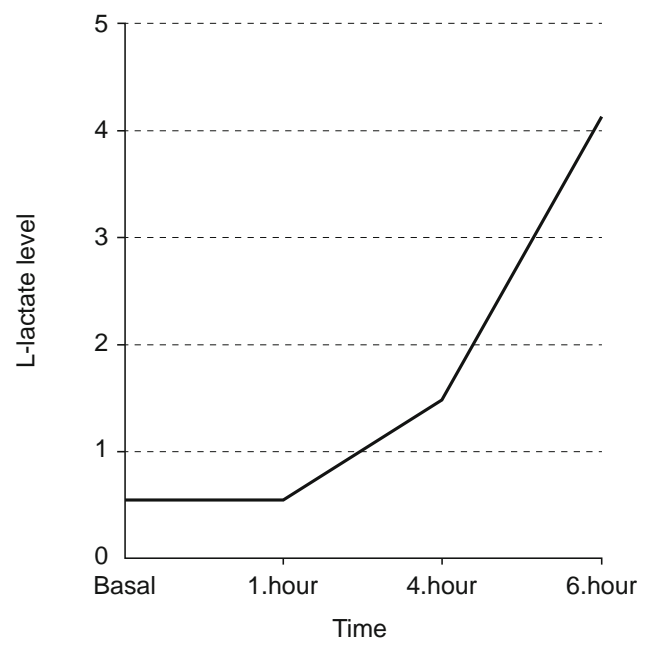

Fig. 7. Post-op L-lactate levels at group III $(p=0.0001$, Friedman variance analyse). Wilcoxon (post hoc): 1st hour L-lactate vs 4th hour $p=$ 0.005 , 4th hour vs 6 th hour $p=0.007$. 


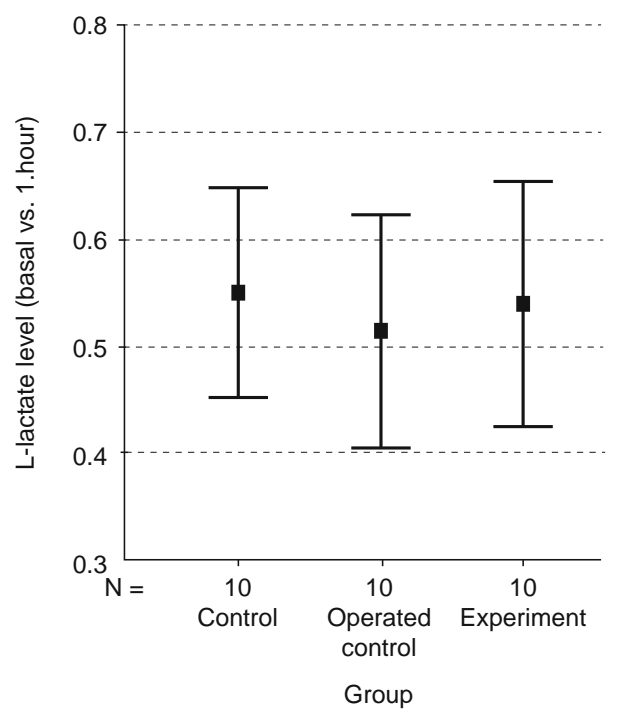

Fig. 8. Post-op 1st hour L-lactate levels and basal L-lactate levels (p $=0.297$, Kruskal-Wallis variance analyse).

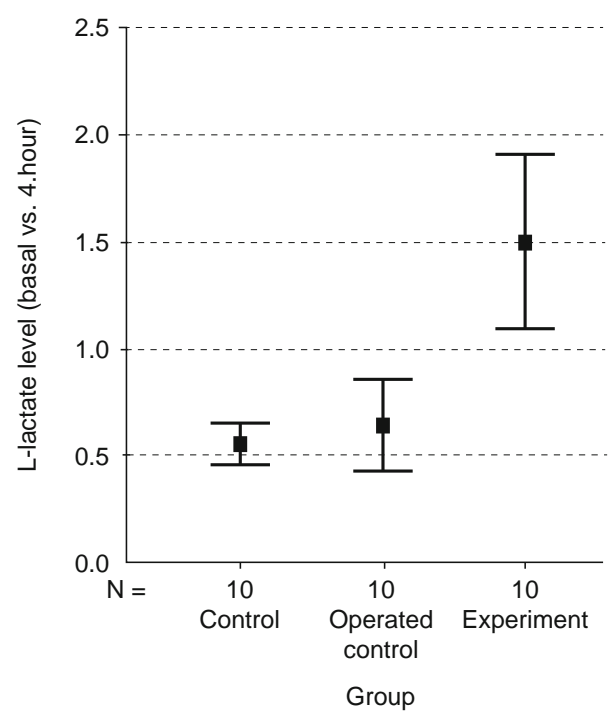

Fig. 9. Post-op 4th hour L-lactate levels and basal L-lactate levels (p $=0.0001$, Kruskal-Wallis variance analyse).

compare postoperative 1, 4, and 6th hour L-lactate levels of groups II and III with basal L-lactate levels of group I. Accordingly, there was no significant difference between L-lactate levels at first hour and basal L-lactate levels $(p=0.297)$ (Fig. 8). In contrast, L-lactate levels at hours 4 and 6 of groups II and III was significantly different from basal L-lactate levels ( $p=0.0001$ vs $p=0.0005$, respectively) (Figs 9 and 10). After lowering the significancy level, paired comparison of L-lactate levels between groups with MannWhitney $U$ test revealed that there was no significant difference between Group II L-lactate levels and 4th hour mean basal levels $(p=0.315)$, but mean L-lactate levels of group III were increased significantly compared to mean basal levels and group II averages ( $p=0.0001$ and $p=0.0002$, respectively). Similarly, there was no

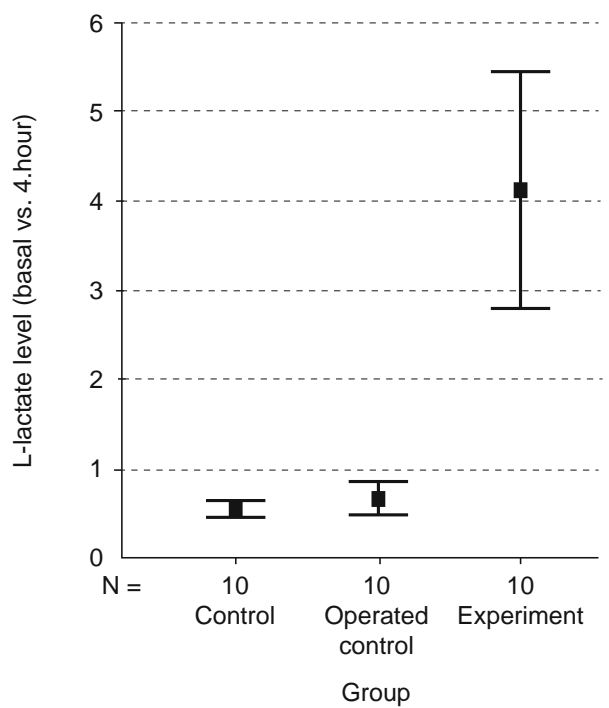

Fig. 10. Post-op 6th hour L-lactate levels and basal L-lactate levels (p $=0.0005$, Kruskal-Wallis variance analyse) .

significant difference between mean basal levels at 6 th hour and group II L-lactate levels $(\mathrm{p}=0.481)$, but mean L-lactate levels of group III were found to be significantly increased compared to basal levels and mean levels of group II ( $\mathrm{p}=0.0001 \mathrm{ad} \mathrm{p}=$ 0.0001 , respectively). Mean basal L-lactate levels of group I and mean L-lactate levels of group II and III at postoperative 1st, 4th and 6th hours are shown in Table 2.

\section{Histopathological assessment}

Intestinal samples gathered from animals with mesenteric ischemia were examined under light microscope following staining process with haematoxylin-eosin. Accordingly, apart from mucosal destruction accompanying transmural tissue necrosis, surface epithelium and intestinal crypts were destructed and villi were totally lost (Fig. 11).

\section{Discussion}

This study shows that blood L-lactate levels of the SMA occluded rats increased significantly either inside the group and in addition when compared to basal values and mean values of opera-

Tab. 2. Mean L-lactate levels.

\begin{tabular}{|c|c|c|c|c|}
\hline L-lactate & $\begin{array}{c}\text { Group I } \\
(\text { mean } \pm \text { SD) }\end{array}$ & $\begin{array}{c}\text { Group II } \\
(\text { mean } \pm \text { SD) }\end{array}$ & $\begin{array}{c}\text { Group III } \\
(\text { mean } \pm \text { SD) }\end{array}$ & $\mathrm{p}$ \\
\hline basal & $0.55 \pm 0.13$ & - & - & \\
\hline 1 st hour & - & $0.51 \pm 0.15$ & $0.53 \pm 0.15$ & $=0.297$ \\
\hline 4th hour & - & $0.63 \pm 0.29$ & $1.49 \pm 0.57$ & $\begin{aligned} & =0.0001 \\
+ & =0.315 \\
* & =0.0001 \\
\S & =0.0002\end{aligned}$ \\
\hline 6th hour & - & $0.67 \pm 0.25$ & $4.12 \pm 1.85$ & $\begin{aligned} & =0.0005 \\
+ & =0.481 \\
* & =0.0001 \\
\S & =0.0001\end{aligned}$ \\
\hline
\end{tabular}




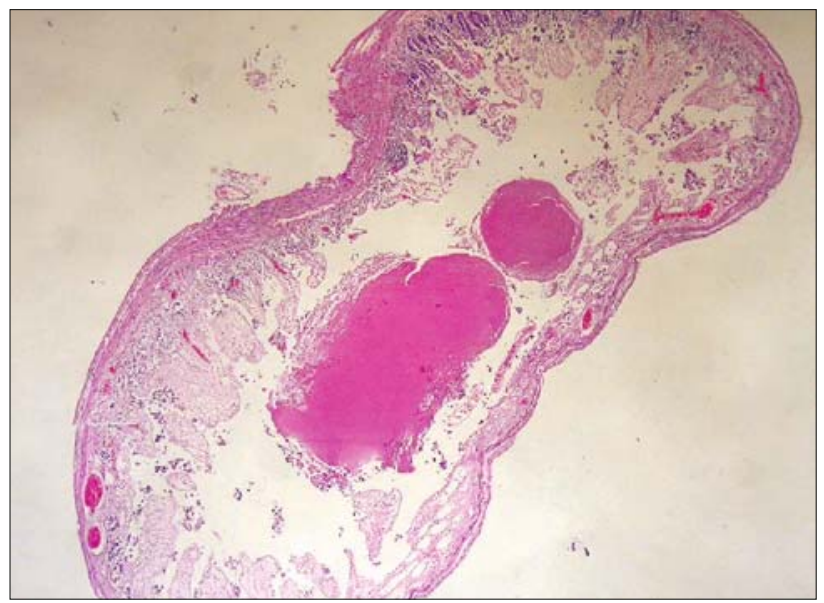

Fig. 11. Mucosal destruction accompanying transmural tissue necrosis, surface epithelium and intestinal crypts were destructed and villi were totally lost.

tive control group starting from 4 th hour of the mesenteric ischemia and this increase continued at 6 th hour. In contrast, serum D-dimer levels did not differ significantly in animals with acute mesenteric ischemia when compared to itself, mean basal levels and 1st, 4th and 6th hour levels of operative control group.

As a fatal disease, incidence of mesenteric ischemia tends to rise, but it constitutes only one in every 1000 hospitalized patients and $1-2 \%$ of hospitalized patients referred with abdominal pain (4). Despite the improvements in medical diagnosis and treatment, mortality rates are around $70 \%(1-4)$. It is clear that this mortality rate is far higher when patients who died before diagnosis are taken into consideration (5). Previous studies showed that only one third of the patients could be diagnosed correctly before death $(5,17)$. In Swedish autopsy studies, it is shown that real mortality rate of mesenteric ischemia can be more than $90 \%$, and $33 \%$ of the patients could be diagnosed before death (8). In many studies it is reported that delays in establishing the AMI diagnosis reduce the survival chance and morbidity significantly $(4,6,18-20)$. In accordance withthis knowledge, with its high mortality and morbidity rates, acute mesenteric ischemia needs rapid and correct diagnosis in order to begin treatment immediately.

Clinical diagnosis of acute mesenteric ischemia is hard to establish, especially in earlier stages of the disease. Findings at the time of referral are usually non-spesific. Nausea and vomiting accompanying to normal abdominal examination usually causes patients to be diagnosed as acute gastroenteritis. Lack of bowel sounds and findings like occult blood in stool does not show up at earlier stages and when found they usually indicate irreversible intestinal damage. There are no specific laboratory tests to exclude acute mesenteric ischemia and especially some of the tests can be deceptive. For example, nearly half of the patients have hyperamylasemia and this can mislead a physician to acute pancreatitis.

Most of the patients referred with acute abdominal pain undergo abdominal X-rays. At earlier stages of the disease, these are rarely diagnostic $(4,21)$. Even though Doppler ultrasonography is useful in complete obstruction of proximal SMA, it is usually used for diagnosis of chronic mesenteric ischemia $(3,4,22)$. Helical computerized tomography and improvements of rapid intravenous contrast agent injection protocols made imaging of the mesenteric vessels easier. Because multi-slice spiral CT and magnetic resonance angiography can create high definition functional images that can show low oxygen saturations, it is promising when compared to conventional CT $(3-5,23,24)$. But these techniques are not widely used to become a standart.

Selective mesenteric angiography is considered golden standart in the diagnosis of mesenteric ischemia (3-6). Studies investigating the place of angiography in AMI diagnosis have shown $90-100 \%$ sensitivity and $100 \%$ specificity $(6,25)$. However, not many hospitals and emergency wards have the opportunity to use angiography in patients with suspected AMI. Moreover, selective mesenteric angiography which is considered a golden standard has many disadvantages including its potential nephrotoxicity, being expensive and a technique that takes time (4-6). Therefore, emergency medicine physicians need faster and simpler methods in AMI suspected patients.

Patients referredto emergency ward with abdominal pain are asked for a series of biochemical laboratory tests. Most frequently encountered laboratory anomalies are leukocytosis and metabolic acidosis (7). Besides, there have been lotof standard enzyme studies including inorganic phosphate and creatinine kinase, lactate dehydrogenase, and aspartate aminotransferase in patients with suspected AMI $(6,14,26)$. Since most of these tests are abnormal in these patients, none of these tests are specific and sensitive enough to make a diagnosis in early stages. Recently, some cytosolic enzymes like glutathione S-transferase and some biochemical parametres located in intestinal mucosal villi like intestinal fatty acid binding protein (I-FABP) are being questioned for usage in AMI as a serological indicator $(27,28)$. Although these studies have promising results, their usage in clinic practice does not seem to be possible and practical. Besides, there are studies about the usage of D-dimer and L-lactateint AMI diagnosis and despite first ones had significant results, especially the latest studies are controversial $(4,10-12,16,29-32)$.

D-dimer is an antigenic product which is the result of a degradation process of cross linked fibrin clot via enzyme plasmin. As an indicator of ongoing thrombus formation and degradation, most common usage area of systemic D-dimer measurements at emergency wards are venous thromboembolic issues like pulmonary embolism and acute deep vein thrombosis and acute coronary syndromes $(33,34)$. Recent studies from Acosta et al. show that D-dimer can be used in patients with suspected AMI for exclusion $(10,29)$. In one of their studies, it is reported that the tests were performed in perioperative period but there is no description about when the tests were exactly performed (29). In their other study, blood was taken in order to analyse D-dimer levels of 101 patients within 24 hours from the onset of abdominal pain and 9 of them were found to be SMA occluded and in these patients median D-dimer levels were elevated significantly (10). In a more recent prospective study, D-dimer was analysed alongside with alpha-glutathion S-transferase, I-FABP, creatinine kinase, lactate dehydrogenase and alkaline phosphatase and only 
D-dimer was found to be relevant with AMI but this test was not specific enough for the diagnosis (13). In experimental studies, D-dimer levels were tested at different time points after SMA occlusion and Altinyollar et al. and Kurt et al. reported significant elevation 30 minutes and 2 hours after ischemia, respectively $(12,30)$. In both studies different animal groups were used for different ischemia periods, so it was not possible to evaluate the changes in D-dimer levels with ongoing ischemia in a specific group. In another study, SMA occluded animals were compared intergroup and with controls following ischemia and it was found that D-dimer levels can't be used as an independent parameter in early AMI diagnosis (16). In our study, with blood taken from a group of SMA occluded animals, D-dimer levels were analysed at 1st, 4th and 6th hour and these results were compared with an operative control group and a control group from whom the basal samples were taken. In addition, evaluations were made among the groups. In conclusion, these comparisons are not showing a significant difference (Figs 1-5, Tab. 1). Elevated D-dimer levels in the studies may be due to time that passed between testing and the onset of pain and existing venous thrombosis. Thus, it is reported in a study that 9 of the test samples were taken at emergency ward and 5 were taken at general surgery ward or intensive care unit (29). In other studies D-dimer tests were made within 24 hours from the onset of abdominal pain, so it is hard to evaluate if this test can be used for early diagnosis $(10,13)$. Previous studies have shown similar results in the meaning of D-dimer elevations in SMA occluded rats, but in our study this elevation was not statistically significant. In addition, different techniques and different monoclonal antibodies are being used at different D-dimer analyses and this can be the cause of encountered variations of results (34). In our study, quantitative assay of D-dimer was made with immunoturbidimetric method. Despite ELISA is being considered a golden standart for D-dimer analyses, immunoturbidimetric analyses are preferable at emergency wards because of their faster and less complex nature and high sensitivity and specivity (34).

Metabolic acidosis is considered an indicator of acute mesenteric ischemia for the last 3 decades (38). Considering the indicators of metabolic acidosis, L-lactate is known to be one of the most practical ways to evaluate laboratory circumstances $(4,31)$. Because mammals only have L-lactate dehydrogenase, pyruvate degradation via this enzyme ends up with L-lactate in cellular hypoxia. Previous clinical studies have shown that lactate levels rise in $90 \%$ of the patients following acute mesenteric ischemia and it is reported that there is a correlation with this elevation and penetration of the ischemia $(4,31)$. In two recent experimental studies, it is shown that blood lactate levels were elevated significantly following mesenteric ischemia $(16,32)$. In contrast, in another experimental study, blood lactate levels failed to achieve a significancy level (11). These researchers came to a decision that lactate might got elevated due to venous stasis at blood sampling. In our research, L-lactate levels were analysed from blood samples of a group of rats taken at 1st, 4 th and 6th hour and results were compared with an operative control group and basal values. In addition, comparisons among the groups were made at given time-points. Accordingly, there was no significant rising tendency in operative control group (Group II) (Fig. 6). However, L-lactate levels of mesenteric ischemia group (Group III, research group) started elevating at 4th hour and this rise continued at 6th hour (Fig. 7). Moreover, when we compared 4th and 6th hour L-lactate levels of groups II and III with basal values, there was a significant difference (Figs 9 and 10). Besides, mean L-lactate levels of group III were found significantly elevated when compared with both mean levels of groups I and II. Similarly, there was no significancy between 6th hour mean basal levels and L-lactate levels of group II, but mean L-lactate levels of group III were found to be significantly elevated when compared to mean basal levels and mean group II levels (Tab. 2).

Blood L-lactate levels were determined from blood taken into capillary tubes with the help of immobilised enzyme electrode technology using YSI 1500 Sport portative lactate analyzer (Yellow Springs Instruments Inc., Ohio-USA). Twenty-five $\mu \mathrm{L}$ blood is enough for the device. Results are acquired within 60 seconds in automatic calibration mode. In a study that compares lactate analysers in the presence of hemoglobine basis oxygen transporters, it is reported that YSI 1500 Sport portative lactate analyser gave the most correct results (39). When the importance of early diagnosis of AMI suspected patients is considered, this method is suitable for usage at emergency setup because it is portative and its giving fast and correct results. Previous clinical studies have reported that lactate levels have $100 \%$ sensitivity but $42 \%$ specificity in mesenteric ischemia early diagnosis $(4,31)$. When all taken together, it is clear that L-lactate levels are important to lead the patients with AMI suspicion to more complex and invasive diagnostic methods.

As a result, in rats with SMA occlusion serum D-dimer levels were not increased significantly when compared either with the group or with the basal values of the control group and values in operative control group. Therefore, it is concluded that D-dimer is not a useful marker at the early diagnosis of AMI. On the other hand, it is revealed that blood L-lactate levels began to increase significantly following 4 th hour of mesenteric ischemia and it is shown that this increase continued at the 6th hour. In addition, considering the utmost importance of the early diagnosis in patients with the clinical suspicion of AMI, L-lactate seems to be a suitable marker to use in emergency departments because it is measured by a portable device that gives fast and accurate results. Nevertheless, our results need to be supported by clinical studies with larger patient series.

\section{References}

1. Menon NJ, Amin AM, Mohammed A, Hamilton G. Acute mesenteric ischaemia. Acta Chir Belg 2005; 105: 344-354.

2. Ujiki M, Kibbe MR. Mesenteric ischemia. Perspect Vasc Surg Endovasc Ther 2005; 17: 309-318.

3. Yasuhara H: Acute mesenteric ischemia: the challenge of gastroenterology. Surg Today 2005; 35: 185-195.

4. Martinez JP, Hogan GJ. Mesenteric ischemia. Emerg Med Clin North Am 2004; 22: 909-928. 
5. Herbert GS, Steele SR: Acute and chronic mesenteric ischemia. Surg Clin North Am 2007; 87: 1115-1134.

6. Kozuch PL, Brandt LJ. Review article: diagnosis and management of mesenteric ischaemia with an emphasis on pharmacotherapy. Aliment Pharmacol Ther 2005; 21: 201-215.

7. Oldenburg WA, Lau LL, Rodenberg TJ, Edmonds HJ, Burger CD. Acute mesenteric ischemia: a clinical review. Arch Intern Med 2004; 164 : 1054-1062.

8. Acosta S, Ogren M, Sternby NH, Bergqvist D, Bjorck M. Incidence of acute thrombo-embolic occlusion of the superior mesenteric artery--a population-based study. Eur J Vasc Endovasc Surg 2004; 27: 145-150.

9. Acosta S, Ogren M, Sternby NH, Bergqvist D, Bjorck M. Clinical implications for the management of acute thromboembolic occlusion of the superior mesenteric artery: autopsy findings in 213 patients. Ann Surg 2005; 241: 516-522.

10. Acosta S, Nilsson TK, Bjorck M. D-dimer testing in patients with suspected acute thromboembolic occlusion of the superior mesenteric artery. Br J Surg 2004; 91: 991-994.

11. Acosta S, Nilsson TK, Malina J, Malina M. L-lactate after embolization of the superior mesenteric artery. J Surg Res 2007; 143: 320-328.

12. Altinyollar H, Boyabatli M, Berberoglu U. D-dimer as a marker for early diagnosis of acute mesenteric ischemia. Thromb Res 2006; 117 : 463-467.

13. Block T, Nilsson TK, Bjorck M, Acosta S. Diagnostic accuracy of plasma biomarkers for intestinal ischaemia. Scand J Clin Lab Invest 2008; 68: $242-248$.

14. Collange O, Tamion F, Chanel S, Hue G, Richard V, Thuilliez C et al. D-lactate is not a reliable marker of gut ischemia-reperfusion in a rat model of supraceliac aortic clamping. Crit Care Med 2006; 34: 1415-1419.

15. Icoz G, Makay O, Sozbilen M, Gurcu B, Caliskan C, Firat O et al. Is D-dimer a predictor of strangulated intestinal hernia? World J Surg 2006; 30: 2165-2169.

16. Kulacoglu H, Kocaerkek Z, Moran M, Kulah B, Atay C, Kulacoglu $\mathrm{S}$ et al. Diagnostic value of blood D-dimer level in acute mesenteric ischaemia in the rat: an experimental study. Asian J Surg 2005; 28: 131-135.

17. Mamode N, Pickford I, Leiberman P. Failure to improve outcome in acute mesenteric ischaemia: seven-year review. Eur J Surg 1999; 165: 203-208.

18. Kougias P, Lau D, El Sayed HF, Zhou W, Huynh TT, Lin PH. Determinants of mortality and treatment outcome following surgical interventions for acute mesenteric ischemia. J Vasc Surg 2007; 46: 467-474.

19. Safioleas MC, Moulakakis KG, Papavassiliou VG, Kontzoglou K, Kostakis A. Acute mesenteric ischaemia, a highly lethal disease with a devastating outcome. Vasa 2006; 35: 106-111.

20. Stamatakos M, Stefanaki C, Mastrokalos D, Arampatzi H, Safioleas $\mathbf{P}$, Chatziconstantinou $\mathbf{C}$ et al. Mesenteric ischemia: still a deadly puzzle for the medical community. Tohoku J Exp Med 2008; 216: 197-204.

21. Umphrey H, Canon CL, Lockhart ME. Differential diagnosis of small bowel ischemia. Radiol Clin North Am 2008; 46: 943-952i.
22. Dietrich CF, Jedrzejczyk M, Ignee A. Sonographic assessment of splanchnic arteries and the bowel wall. Eur J Radiol 2007; 64: 202-212.

23. Horton KM, Fishman EK. Multidetector CT angiography in the diagnosis of mesenteric ischemia. Radiol Clin North Am 2007; 45: 275-288.

24. Smith CL, Horton KM, Fishman EK. Mesenteric CT angiography: a discussion of techniques and selected applications. Tech Vasc Interv Radiol 2006; 9: 150-155.

25. Brandt LJ, Boley SJ.AGA technical review on intestinal ischemia. American Gastrointestinal Association. Gastroenterology 2000; 118: 954-968.

26. Berland T, Oldenburg WA. Acute mesenteric ischemia. Curr Gastroenterol Rep 2008; 10: 341-346.

27. Gearhart SL, Delaney CP, Senagore AJ, Banbury MK, Remzi FH, Kiran RP et al. Prospective assessment of the predictive value of alphaglutathione S-transferase for intestinal ischemia. Am Surg 2003; 69: 324329; discussion 329.

28. Guthmann F, Borchers T, Wolfrum C, Wustrack T, Bartholomaus S, Spener F. Plasma concentration of intestinal- and liver-FABP in neonates suffering from necrotizing enterocolitis and in healthy preterm neonates. Mol Cell Biochem 2002; 239: 227-234.

29. Acosta S, Nilsson TK, Bjorck M. Preliminary study of D-dimer as a possible marker of acute bowel ischaemia. Br J Surg 2001; 88: 385-388.

30. Kurt Y, Akin ML, Demirbas S, Uluutku AH, Gulderen M, Avsar K et al. D-dimer in the early diagnosis of acute mesenteric ischemia secondary to arterial occlusion in rats. Eur Surg Res 2005; 37: 216-219.

31. Lange H, Jackel R. Usefulness of plasma lactate concentration in the diagnosis of acute abdominal disease. Eur J Surg 1994; 160: 381-384.

32. Kurimoto Y, Kawaharada N, Ito T, Morikawa M, Higami T, Asai Y. An experimental evaluation of the lactate concentration following mesenteric ischemia. Surg Today 2008; 38: 926-930.

33. Siragusa S. D-dimer testing: advantages and limitations in emergency medicine for managing acute venous thromboembolism. Intern Emerg Med 2006; 1: 59-66.

34. Wakai A, Gleeson A, Winter D. Role of fibrin D-dimer testing in emergency medicine. Emerg Med J 2003; 20: 319-325.

35. Danesh J, Whincup P, Walker M, Lennon L, Thomson A, Appleby P et al. Fibrin D-dimer and coronary heart disease: prospective study and meta-analysis. Circulation 2001; 103: 2323-2327.

36. Fujii Y, Takeuchi S, Harada A, Abe H, Sasaki O, Tanaka R. Hemostatic activation in spontaneous intracerebral hemorrhage. Stroke 2001; 32: $883-890$.

37. Quick G, Eisenberg P. Bedside measurement of D-dimer in the identification of bacteremia in the emergency department. J Emerg Med 2000; 19: $217-223$.

38. Brooks DH, Carey LC. Base deficit in superior mesenteric artery occlusion, an aid to early diagnosis. Ann Surg 1973; 177: 352-356.

39. Jahr JS, Osgood S, Rothenberg SJ, Li QL, Butch AW, Gunther R et al. Lactate measurement interference by hemoglobin-based oxygen carriers (Oxyglobin, Hemopure, and Hemolink). Anesth Analg 2005; 100: 431-436. 